\title{
Risk of ischaemic stroke in patients with transient global amnesia: a propensity-matched cohort study
}

\author{
Sang Hum Lee (1) , ${ }^{1}$ Keon-Yeup Kim, ${ }^{1}$ Jeong-Woo Lee, ${ }^{2}$ So-Jeong Park, ${ }^{2}$ \\ Jin-Man Jung (D) ${ }^{1,3}$
}

To cite: Lee SH, Kim K-Y, Lee J-W, et al. Risk of ischaemic stroke in patients with transient global amnesia: a propensitymatched cohort study. Stroke \& Vascular Neurology 2021;0. doi:10.1136/svn-2021-001006

Received 16 March 2021 Accepted 3 October 2021
Check for updates

(C) Author(s) (or their employer(s)) 2021. Re-use permitted under CC BY-NC. No commercial re-use. See rights and permissions. Published by BMJ.

${ }^{1}$ Department of Neurology, Korea University Ansan Hospital, Korea University College of Medicine, Ansan, Republic of Korea

${ }^{2}$ Data Science Team, Hanmi Pharm. Co., Ltd, Seoul, Republic of Korea

${ }^{3}$ Korea University Zebrafish Translational Medical Research Center, Ansan, Republic of Korea

Correspondence to

Dr Jin-Man Jung;

dr.jinmanjung@gmail.com

\section{ABSTRACT}

Background The exact pathophysiological mechanism of transient global amnesia (TGA) is unknown. It is debatable whether TGA is a risk factor for stroke. Therefore, here we investigated the possibility of TGA as a risk factor for stroke in a real-world setting using large-scale nationwide health claims data.

Methods We used health claims data from the Korean National Health Insurance Service (NHIS). Patients diagnosed with TGA between 2007 and 2013 were selected. We initially extracted patients without TGA who were preferentially matched for age and sex with the patients with TGA at a ratio of 10:1 from the whole dataset. Further, we performed 1:2 propensity score matching analysis to balance the baseline characteristics between the two groups. In the propensity score-matched dataset, we performed multivariable Cox regression analysis to investigate the association between TGA and stroke type, including ischaemic, haemorrhagic and all stroke types.

Results Patients with TGA ( $n=14$ 673) were selected from the NHIS database. After extracting from the whole database ( $n=140486)$ and propensity score matching their data at a 1:2 ratio, a total of 10448 and 20442 patients were finally assigned to the TGA and control groups, respectively. The multivariable Cox regression analysis demonstrated that the TGA group had a higher risk of ischaemic stroke and all types of stroke (adjusted $\mathrm{HR}=1.194 ; 95 \% \mathrm{Cl}: 1.043$ to 1.368 ; and $\mathrm{HR}=1.197 ; 95 \%$ Cl: 1.056 to 1.357 , respectively).

Conclusions Analysis of the nationwide claims database showed that TGA could be an important risk factor for stroke, especially for ischaemic stroke.

\section{INTRODUCTION}

The pathological deterioration of memory is known as amnesia. ${ }^{12}$ Transient global amnesia (TGA) is characterised by the sudden onset of anterograde amnesia lasting up to 24 hours. ${ }^{3}$ Since its initial description, various pathophysiological mechanisms have been proposed to explain the aetiology of TGA. One such accepted hypothesis is cortical spreading depression similar to that occurring in migraines. ${ }^{4}$ Other hypotheses include arterial ischaemia and epilepsy-related causes. ${ }^{56}$ However, the exact pathophysiological mechanism of TGA is unknown. ${ }^{7}$
Because of the possible pathophysiology originating from the ischaemia and transient features of TGA, previous studies have reported varying degrees of association between stroke or transient ischaemic attack (TIA) and TGA episodes; however, it is debatable whether TGA is a risk factor for stroke. ${ }^{8-13}$ Therefore, this study aimed to evaluate the long-term patient prognosis after TGA and the potential use of TGA as a potential risk factor for stroke in a real-world setting using large-scale nationwide health claims data from South Korea.

\section{METHODS}

\section{Data sources}

We used health claims data from the Korean National Health Insurance Service (NHIS). All Koreans are eligible for and required to have universal health coverage under the NHIS. The NHIS database records the diagnosis at each hospital visit according to the 10th revision of the International Statistical Classification of Diseases (ICD-10) and includes data on health insurance claims; prescriptions; medical and pharmaceutical records (name, duration, date and dose); hospital admissions; medical procedures; clinical diagnoses; clinical characteristics such as age, sex and personal information (eg, household income); and death statistics of all subscribers. ${ }^{14-16}$

The Korean NHIS database includes both inpatient and outpatient information. The availability of inpatient and outpatient information depends on the application aspects and situation of specific diseases. For specific outcomes, including ischaemic and haemorrhagic stroke, inpatient data were used to clearly verify the outcome event; however, risk factors such as comorbidities such as seizure, TIA and migraine were considered regardless of hospitalisation.

The ICD-10 coding system was implemented in January 1995 in Korea, and its 


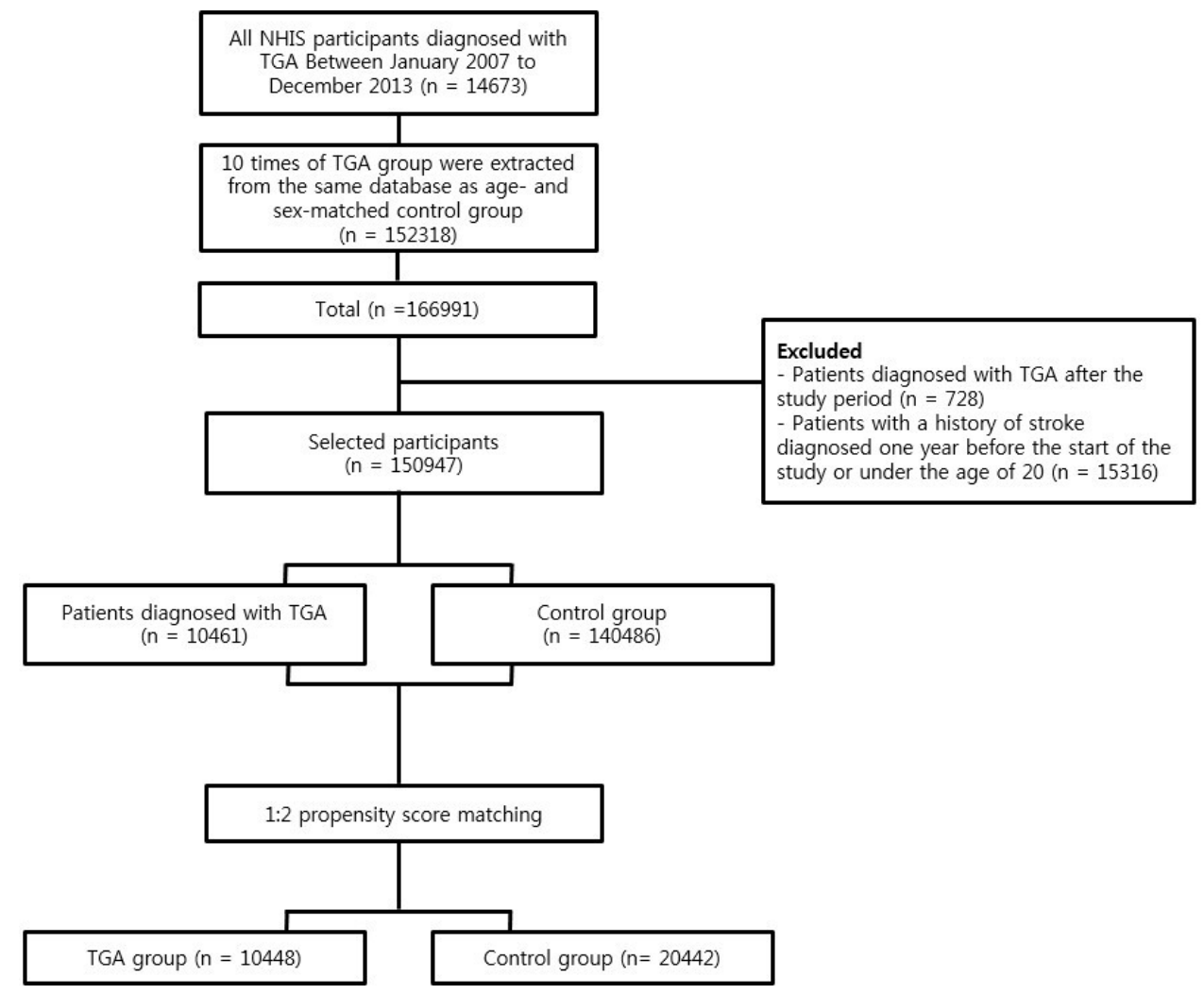

Figure 1 Flow chart for the selection of cases and controls based on a propensity score-matched cohort study of the National Health Insurance Service (NHIS) data. TGA, transient global amnesia.

terminology was revised in January 2003 and used today. Since our study period started in 2005 and included a washout period of 2 years, the code, disease name, and disease category information did not change during the study period and the data were extracted from the same system.

\section{Patient selection and covariates definition}

A flow chart of the patient selection process is shown in figure 1. Patients diagnosed with TGA (code: G45.4) between January 2007 and December 2013 were selected from the NHIS database. Additional factors, including brain diffusion-weighted imaging, were not included in the TGA diagnosis since they were not appropriate for this nationwide dataset, as the detection rate of hyperintense lesions on diffusion-weighted imaging in TGA is relatively low $(30 \%)$ and specific imaging factors such as magnetic field strength $3 \mathrm{~T}$, b value $2000 \mathrm{~s} / \mathrm{mm}^{2}$ and thin-slice sequence $\leq 3 \mathrm{~mm}$ could increase the detection rate. ${ }^{17}$ The index date of the cohort was defined as the date of the hospital visit irrespective of outpatient and inpatient management. A 10-fold larger TGA group was extracted from the same database as the age-matched and sex-matched groups. Patients diagnosed with TGA after the study period were excluded. Patients diagnosed with stroke within a year before the start of the study or the determination of TGA were also excluded to ensure a washout period and reduce the possibility of confusion with ischaemic amnesia. ${ }^{18}$ Patients aged $<20$ years were also excluded. The case and age-matched and sexmatched extraction groups were followed up until the development of the outcome of interest or the end date of the study (31 December 2018).

Baseline data included sex; age; TGA aetiological or mimicking diseases including seizure/epilepsy, depressive disorder, anxiety and migraine; vascular risk factors such as hypertension, diabetes mellitus (DM), hyperlipidaemia and atrial fibrillation (A-Fib); and pre-existing cardiovascular disease including myocardial infarction (MI) or antithrombotic medication use. Hypertension was considered relevant only if the participant was diagnosed with a certain ICD-10 code (I10-13, I15) before or during hospitalisation; DM was defined as the presence of ICD-10 codes E10-14, and hyperlipidaemia was defined as the presence of ICD-10 code E78. ${ }^{19}$ In the subsequent follow-up, all ICD-10 codes for which the diagnosis was excluded were removed. The presence of A-Fib (I48), MI (I21), seizure/ epilepsy (G40, G41, R56), depressive mood disorder (F32, F34), anxiety (F41), and migraine (G43) was defined by the presence of the relevant ICD-10 diagnostic codes in the NHIS records before or during the hospitalisation for stroke. Antithrombotic agents, including oral antiplatelet agents and anticoagulants (aspirin, clopidogrel, triflusal, ticlopidine, cilostazol, ticagrelor, prasugrel, warfarin, apixaban, dabigatran and rivaroxaban), were evaluated based on prescription records. Edoxaban was not available in South Korea during the study period. 


\section{Validation of the ICD-10 code for TGA}

To validate the ICD-10 code for TGA (G45.4), we retrospectively reviewed the medical records at our institution to confirm its sensitivity and specificity. The validation was conducted based on the method used in a previous study. ${ }^{20}$ We identified 30 patients diagnosed with TGA between 2007 and 2014 with a primary ICD-10 discharge diagnosis of G45.4 and 30 patients with other primary cerebrovascular disease codes (eg, TIA) without the TGA code. A neurologist (JYK) who was blinded to the patients' ICD-10 diagnostic codes reviewed the medical records and adjudicated the cases of TGA based on its standard definition of the sudden onset of anterograde and retrograde memory lasting up to 24 hours without other accompanying neurological deficits. ${ }^{21}$ We used his (JYK) clinical decision about the primary diagnosis as the gold standard and calculated the sensitivity and specificity of the ICD-10 code for TGA (G45.4).

\section{Outcomes of interest and measures}

The outcomes of interest were ischaemic stroke (IS) (I63), intracerebral haemorrhage (ICH) (I61) and all types of stroke (I60-63), including IS, ICH, subarachnoid haemorrhage (SAH) (I60) and subdural haemorrhage (I62) accompanied by hospitalisation. Each participant was followed up from the index date to the earliest occurrence of any study outcome or the end of the study period (31 December 2018).

\section{Statistical analysis}

The data are presented as mean (SD) for continuous variables and number (percentage) for categorical variables. The $\chi^{2}$ test was used to analyse intergroup differences. Differences in continuous variables were assessed using Student's t-test.

We performed propensity score matching to balance the baseline characteristics between the case and control groups. The use of a propensity score with time-to-event data using weighting or matching techniques is well established..$^{22}$ A propensity score was calculated for each patient based on the key baseline covariates. Propensity score matching (maximum 1:2) was performed for patients diagnosed with TGA. The propensity score was built using a multivariable logistic regression model considering the following variables: sex, age, seizure/epilepsy, depressive disorder, anxiety, migraine, hypertension, DM, hyperlipidaemia, MI, A-Fib and antithrombotic medication. We used greedy nearest neighbour matching on the logit of the propensity score using callipers with a width of 0.1 of the SD of the logit of the propensity score.

In the propensity score-matched dataset, we performed multivariable Cox regression analysis adjusted only by enrolment year to investigate the association between TGA and stroke type. In contrast with the control group, the TGA group was diagnosed during the study period; thus, patients diagnosed with TGA in the middle or late study period had a relatively short follow-up period. Therefore, enrolment year was considered in the computation of the adjusted HRs. Accounting for death as a competing risk, the proportional hazards model for the subdistribution using cause-specific Cox regression was applied. The results were considered statistically significant when the $p$ value was $<0.05$. All statistical analyses and propensity score matching were performed using SAS software (V.9.4; SAS Institute,).

\section{Standard protocol approvals, registrations and patient consent}

The NHIS data were fully anonymised to protect patient privacy.

\section{RESULTS}

Patients diagnosed with TGA ( $\mathrm{n}=14673$ ) between January 2007 and December 2013 were initially selected from the NHIS database. After the application of the 10-fold control group extraction process and the exclusion criteria process, 10461 (6.93\%) patients were assigned to the TGA group vs 140486 (93.07\%) to the control group. After 1:2 propensity score matching, a total of 10448 and 20442 patients were finally assigned to the TGA and control groups, respectively (figure 1). The mean (SD) follow-up period was $10.00(2.76)$ years.

Based on the validation of the medical record review, the ICD-10 code G45.4 had a sensitivity of $100 \%(95 \%$ CI: $0.86 \%$ to $1.00 \%$ ) and a specificity of $83.3 \%$ (95\% CI: $0.74 \%$ to $0.83 \%$ ) for TGA.

Table 1 shows the demographic characteristics of the TGA and age-matched and sex-matched extraction groups. The TGA group had a higher incidence of hypertension; hyperlipidaemia; cardiac disease; TGA aetiological or mimicking diseases, including depressive disorder, anxiety, seizure/epilepsy and migraine; and antithrombotic users than the age-matched and sex-matched extraction group. Compared with absolute standardised differences noted before the propensity score matching (TGA case vs age-matched and sex-matched extraction group), after propensity score matching, all absolute standardised differences were less than 0.1. Figure 2 shows that all baseline variables were well balanced between the TGA and control groups.

Table 2 shows the crude and adjusted HRs for all outcomes. After adjustment for the enrolment year, participants with TGA had a higher risk of IS and all types of stroke (adjusted HR (aHR) $=1.194 ; 95 \%$ CI: 1.043 to 1.368; and aHR=1.197; 95\% CI: 1.056 to 1.357 , respectively). There were no significant intergroup differences in haemorrhagic stroke subtypes, such as SAH, ICH and subdural haematoma (aHR $=1.104 ; 95 \%$ CI: 0.665 to 1.832 ; aHR $=0.845 ; 95 \%$ CI: 0.606 to 1.177 and $\mathrm{aHR}=1.849 ; 95 \%$ CI: 0.997 to 3.430 , respectively)

\section{Predictors for IS and all stroke types}

Patients with TGA with hypertension, DM and A-Fib had a higher risk of IS (aHR=1.545, 95\% CI: 1.266 to 1.885 , $\mathrm{p}<0.001 ; \mathrm{aHR}=1.321,95 \%$ CI: 1.116 to $1.565, \mathrm{p}=0.001$; aHR $=1.564,95 \%$ CI: 1.106 to 2.213 , $\mathrm{p}=0.011$, respectively), 
Table 1 Baseline characteristics of the TGA cases versus age-matched and sex-matched extraction group and absolute standardised differences after propensity score matching

\begin{tabular}{|c|c|c|c|c|}
\hline \multirow[b]{2}{*}{ Clinical values } & \multirow[b]{2}{*}{$\begin{array}{l}\text { TGA case } \\
(n=10461)\end{array}$} & \multirow{2}{*}{$\begin{array}{l}\text { Age-matched } \\
\text { and sex-matched } \\
\text { extraction group } \\
(n=140486)\end{array}$} & \multicolumn{2}{|c|}{ Absolute standardised difference } \\
\hline & & & $\begin{array}{l}\text { Before PS } \\
\text { matching }\end{array}$ & $\begin{array}{l}\text { After 1:2 PS matching } \\
\text { (TGA, } n=10448 \text { ) } \\
\text { (control, } n=20442 \text { ) }\end{array}$ \\
\hline Age (years) & $60.5 \pm 12.6$ & $60.7 \pm 12.4$ & 0.018 & 0.032 \\
\hline Female sex & $7067(67.6)$ & $93117(66.3)$ & 0.027 & 0.013 \\
\hline \multicolumn{5}{|l|}{ Stroke risk factors } \\
\hline Hypertension & $5161(49.3)$ & $53017(37.7)$ & $0.236^{\star}$ & 0.018 \\
\hline DM & $3772(36.1)$ & $29933(21.3)$ & $0.331^{*}$ & 0.025 \\
\hline Hyperlipidaemia & $5815(55.6)$ & 32462 (23.1) & $0.705^{\star}$ & 0.014 \\
\hline \multicolumn{5}{|l|}{ Cardiac disease } \\
\hline A-Fib & $263(2.5)$ & $1727(1.2)$ & 0.095 & 0.009 \\
\hline MI & $262(2.5)$ & $1536(1.1)$ & $0.106^{\star}$ & 0.016 \\
\hline Depressive d/o & $396(3.8)$ & $1655(1.2)$ & $0.168^{*}$ & 0.051 \\
\hline Anxiety $\mathrm{d} / \mathrm{o}$ & $3858(36.9)$ & $18217(13.0)$ & $0.575^{\star}$ & 0.045 \\
\hline Seizure/epilepsy & $73(0.7)$ & $528(0.4)$ & 0.044 & 0.005 \\
\hline Migraine & $1908(18.2)$ & $8397(6.0)$ & $0.382^{*}$ & 0.069 \\
\hline Antithrombotic user & $8383(80.1)$ & $67108(47.8)$ & $0.716^{\star}$ & 0.009 \\
\hline
\end{tabular}

Results are expressed as number (\%) or mean \pm SD. Antithrombotics include antiplatelet agents and anticoagulants.

*Absolute standardised difference $\geq 0.1$.

A-Fib, atrial fibrillation; DM, diabetes mellitus; d/o, disorder; MI, myocardial infarction; PS, propensity score; TGA, transient global amnesia.

after adjusting for confounding factors such as age, sex and comorbidities (table 3). Consistent with IS, hypertension, DM and A-Fib were associated with an increased risk of all stroke types $(\mathrm{aHR}=1.446$; $95 \%$ CI: 1.206 to 1.732 ; $\mathrm{p}<0.001 ; \mathrm{aHR}=1.252 ; 95 \%$ CI: 1.070 to $1.464 ; \mathrm{p}=0.005$; aHR=1.600; 95\% CI: 1.156 to $2.215 ; \mathrm{p}=0.004$, respectively) (table 3).

\section{DISCUSSION}

Here, we investigated the relationship between TGA and stroke type using a propensity score-matched cohort from nationwide health insurance claims data. Using these

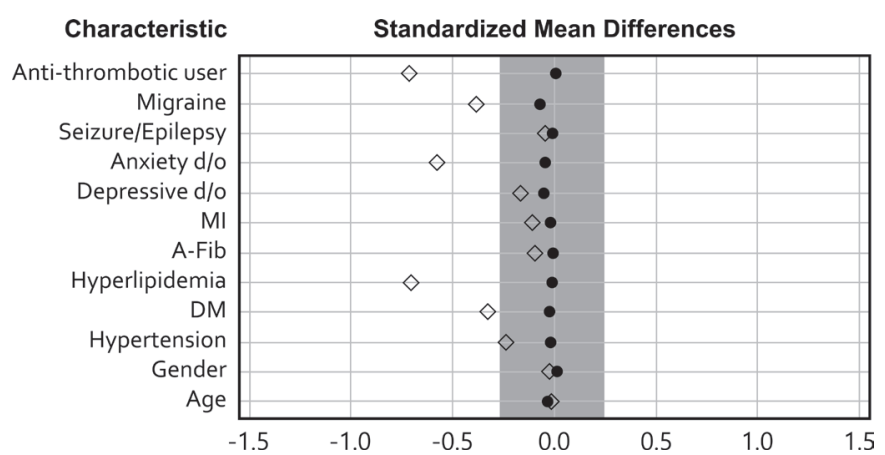

Figure 2 Standardised differences in variables included in the propensity score. Black lozenges indicate the entire study population (140 478 participants). Black dots indicate the propensity score-matched participants (20 493 participants). A-Fib, atrial fibrillation; DM, diabetes mellitus; d/o, disorder; MI, myocardial infarction. data, we evaluated long-term and real-world patient prognosis after TGA, especially focusing on the occurrence of stroke. TGA was a risk factor for stroke, particularly IS, but not haemorrhagic stroke. The risk of IS is increased in patients with TGA with hypertension, DM and A-Fib.

Our results are consistent with those of several previous studies prior to the 1980s suggesting that TGA could cause stroke. ${ }^{1-1323}$ In contrast, modern studies have reported no association between TGA and stroke. ${ }^{8-10202425}$ TGA is reportedly less likely to be a risk factor for stroke and does not predict stroke compared with TIA, seizure, migraine or normal controls. However, these recent studies were diverse in terms of study population and design, subjects' characteristics and follow-up period. Compared with most studies of hospital-based datasets, Garg et al and Mangla et al used a relatively large nationwide database or administrative claims data, ${ }^{2025}$ while Romoli et al used a combination of a population-based dataset and a hospital-based registry (number of patients with TGA=21 202, 4299 and 525 , respectively), ${ }^{24}$ but many other studies had small sample sizes of patients with TGA $(<200)$. These three studies showed a negative association; however, the short ( $<1$ year) follow-up period in the Garg et al and Mangla et al studies did not enable investigation of the long-term effect of TGA. ${ }^{20} 25$

Romoli et al's study results appear non-generalisable in that all cardiovascular risk factors tended to be treated in this cohort, which could contribute to the very low stroke risk during long-term follow-up. Study design is also variable, ranging from case series to case-control studies 
Table 2 Multivariable analysis of outcomes among TGA participants versus propensity score-matched control participants

\begin{tabular}{|c|c|c|c|c|}
\hline Variables & Crude HR (95\% Cl) & $P$ value & Adjusted HR $(95 \% \mathrm{Cl})^{*}$ & $P$ value \\
\hline Ischaemic stroke & 0.916 (0.846 to 0.992$)$ & 0.032 & $1.194(1.043$ to 1.368$)$ & 0.010 \\
\hline \multicolumn{5}{|c|}{ Haemorrhagic stroke } \\
\hline $\mathrm{ICH}$ & $0.943(0.764$ to 1.163$)$ & 0.581 & 0.845 (0.606 to 1.177$)$ & 0.319 \\
\hline $\mathrm{SDH}$ & 1.684 (1.172 to 2.421$)$ & 0.005 & 1.849 (0.997 to 3.430$)$ & 0.051 \\
\hline
\end{tabular}

Total number of propensity score-matched participants, $n=150947$ (10 461 patients with TGA and 140486 control participants). Multivariable Cox regression analysis was used to examine the diagnoses of TGA and all-type stroke including ICH, SDH, SAH and ischaemic stroke. *Adjusted for enrolment year.

$\mathrm{ICH}$, intracerebral haemorrhage; SAH, subarachnoid haemorrhage; SDH, subdural haematoma; TGA, transient global amnesia.

and retrospective or prospective cohorts with or without propensity score matching. In addition, most studies evaluating the development of stroke after TGA have been conducted in Western populations. ${ }^{8-102025}$

Vascular risk factor profiles differed between patients with TGA and TIA and normal controls. ${ }^{1012}$ One hospitalbased study showed a difference in sex ratio and cardiovascular risk factors in the Asian TGA group compared with previous studies. ${ }^{7}$ With regard to the negative association reported by one propensity score matching study that could minimise the imbalance in baseline cardiovascular risk factors between TGA and control groups, careful interpretation is needed due to the relatively short follow-up period (approximately 6 months). ${ }^{25}$ The intrastudy limitations and interstudy diversity might contribute to the inconsistent association between TGA and subsequent stroke. This could have affected the decrease in specificity, as was the case with a large sample dataset from claims data. ${ }^{20}$ However, our study was performed in a real-world setting using large-scale nationwide health claims data from the NHIS. In addition, propensity score matching based on key baseline covariates as well as psychogenic aspects of TGA, such as depressive disorder, anxiety disorder, and neurophysiological problems such as seizure/epilepsy and migraine as signs of causes or symptoms, was performed in a database of approximately 150000 patients. Finally, approximately 10000 patients diagnosed with TGA were enrolled in this largescale study, which had the advantage of a long (10-year) follow-up period.

TGA, migraine (migraine with aura, migraine amnesia), epileptic seizures (transient epileptic amnesia), dissociative amnesia, depression, anxiety and limbic encephalitis may cause acute amnesia. ${ }^{126}$ It is controversial and still inconclusive whether vascular risk factors can be a clue to identifying and differentiating TGA cases. Some previous studies noted that such risk factors were hardly distinguishable between TGA and mimicking diseases, and TGA did not show a particularly distinct increase in risk factors or a clear association compared with other mimics. ${ }^{202} 28$ Instead, Romoli et al reported that clinical criteria rather vascular risk factors were likely effective for TGA diagnosis. ${ }^{28}$ Our study using age-matched and sex-matched datasets demonstrated that vascular risk factors including hypertension, hyperlipidaemia, and cardiac diseases such as A-Fib and MI were significantly higher in the TGA group; these comorbidities sustain a risk factor for IS in the multivariable analysis after the adjustment for confounding factors. This could imply that if a patient presenting with acute amnesia had accompanying vascular risk factors, the possibility of TGA rather than other causes should be preferentially considered, which is consistent with previous results. ${ }^{7}$ In addition, the higher prevalence of conventional risk factors (DM, hypertension and A-Fib) can be attributed to the development of IS in TGA, although patients with TGA showed a lower prevalence of vascular risk factors than patients with TIA and lacunar cerebral infarction. ${ }^{29}$ However, this paradigm shift could potentially bias the TGA diagnosis since it is based on clinical criteria, not on concomitant cardiovascular risk factors. Rather, our conclusion might better be applied to patients with TGA with several risk factors who are already taking antithrombotics $(81 \%)$ despite our results being extracted from real-world data. Also, vascular risk factors in patients with amnesia are not negligible because the under-recognition of ischaemic amnesia as a TGA mimic may lead to alternative treatment, therefore representing a condition not to be missed.

Our study had several notable limitations. First, as detailed clinical information was not acquired from the health claims data, it was not possible to assess blood pressure, hyperlipidaemia, DM control, stroke severity or imaging findings for making the stroke diagnosis, which may be an important determinant of stroke. Second, although we were able to access the patients' entire prescription history, there may have been differences between the prescription and the patient's actual compliance with the medication. Third, the determination of TGA, comorbidities and results were based on the diagnostic code in the health claims records. As our analysis mainly relied on administrative data and ICD-10 diagnostic codes without detailed clinical, supportive imaging and laboratory data, the results from these claims data may be inaccurate or biased by nature despite efforts to reduce such possibilities. Fourth, when validation was 


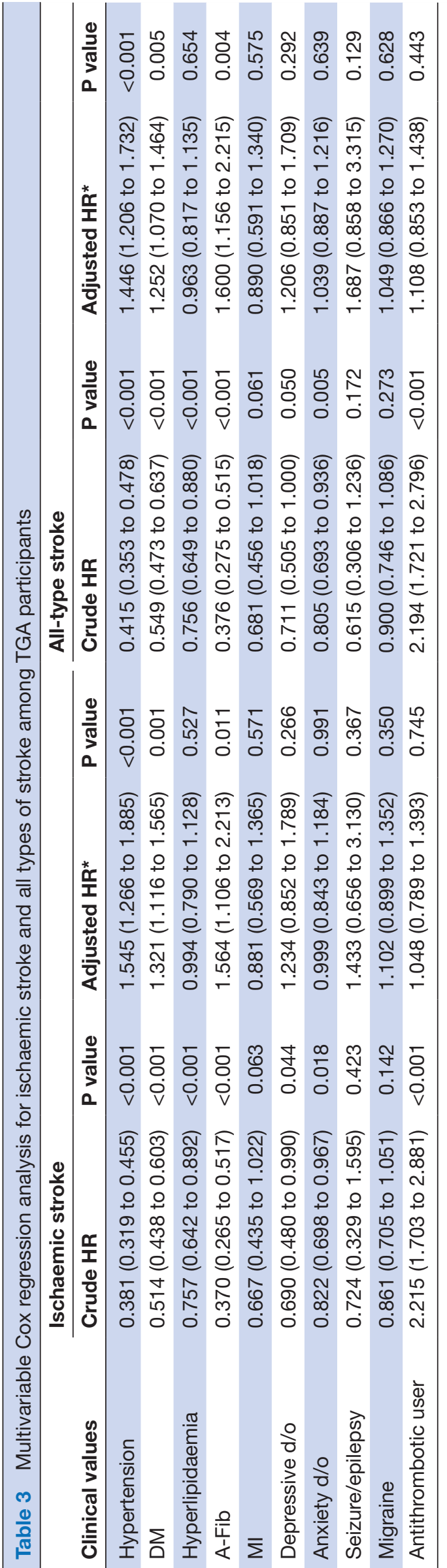

performed of the medical records investigated by our institution, the specificity was approximately $83.3 \%$. This is probably because we compared health claims data using the ICD-10 code with hospital data confirmed through individualised clinical information. The TGA group in our study may have included some mimicking diseases or be vulnerable to inaccurate or biased ascertainment. This possibility could have affected the decrease in specificity, as was the case with a large sample dataset of claims data. ${ }^{20}$

In conclusion, this study based on real-world data from South Korea demonstrated that TGA can be an important risk factor for stroke, especially IS. Among patients with TGA, those who had specific conventional risk factors, such as hypertension, DM and A-Fib, were at a higher risk of developing IS. Further well-designed and large studies are warranted to confirm these findings.

Contributors SHL contributed to the study conception and design, data analysis, acquisition of clinical and imaging data, statistical analysis, manuscript drafting and revision. J-MJ contributed to the study conception and design, analysis and interpretation of the imaging and clinical data, manuscript drafting and revision, and study supervision and J-MJ acts as guarantor. K-YK contributed to the data analysis and manuscript revision. J-WL and S-JP contributed to the data statistical analysis.

Funding This study was supported by the Basic Science Research Program through the National Research Foundation of Korea (NRF) funded by the Ministry of Science and ICT (NRF-2020R1C1C1009294) and Korea University Grant.

Competing interests J-MJ has received the following: lecture honoraria from Pfizer, Sanofi-Aventis, Otsuka, and Hanmi Pharmaceutical Co; and consulting fees from Daewoong Pharmaceutical Co.

Patient consent for publication Not required.

Ethics approval This study obtained ethics approval from the Institutional Review Board of Korea University Ansan Hospital (IRB no. 2019AS0027). This study was approved and the requirement for informed consent was waived by the Institutional Review Board of Korea University Ansan Hospital.

Provenance and peer review Not commissioned; externally peer reviewed. Data availability statement All data relevant to the study are included in the article or uploaded as supplemental information.

Open access This is an open access article distributed in accordance with the Creative Commons Attribution Non Commercial (CC BY-NC 4.0) license, which permits others to distribute, remix, adapt, build upon this work non-commercially, and license their derivative works on different terms, provided the original work is properly cited, appropriate credit is given, any changes made indicated, and the use is non-commercial. See: http://creativecommons.org/licenses/by-nc/4.0/.

ORCID iDs

Sang Hum Lee http://orcid.org/0000-0002-9538-446X

Jin-Man Jung http://orcid.org/0000-0003-0557-6431

\section{REFERENCES}

1 Bartsch T, Butler C. Transient amnesic syndromes. Nat Rev Neurol 2013;9:86-97.

2 Markowitsch HJ, Staniloiu A. Amnesic disorders. Lancet 2012;380:1429-40.

3 Arena JE, Rabinstein AA. Transient global amnesia. Mayo Clin Proc 2015;90:264-72.

4 Caplan L, Chedru F, Lhermitte F, et al. Transient global amnesia and migraine. Neurology 1981;31:1167-70.

5 Di Filippo M, Calabresi P. Ischemic bilateral hippocampal dysfunction during transient global amnesia. Neurology 2007;69:493.

6 Jacome DE. Eeg features in transient global amnesia. Clin Electroencephalogr 1989;20:183-92.

7 Jang J-W, Park SY, Hong J-H, et al. Different risk factor profiles between transient global amnesia and transient ischemic attack: a large case-control study. Eur Neurol 2014;71:19-24.

8 Zorzon M, Antonutti L, Masè G, et al. Transient global amnesia and transient ischemic attack. natural history, vascular risk factors, and associated conditions. Stroke 1995;26:1536-42. 
9 Gandolfo C, Caponnetto C, Conti M, et al. Prognosis of transient global amnesia: a long-term follow-up study. Eur Neurol 1992;32:52-7.

10 Pantoni L, Bertini E, Lamassa $\mathrm{M}$, et al. Clinical features, risk factors, and prognosis in transient global amnesia: a follow-up study. Eur $J$ Neurol 2005;12:350-6.

11 Jensen TS, De Fine Olivarius B. Transient global amnesia as a manifestation of transient cerebral ischemia. Acta Neurol Scand 1980;61:115-24.

12 Guidotti M, Anzalone N, Morabito A, et al. A case-control study of transient global amnesia. J Neurol Neurosurg Psychiatry 1989;52:320-3.

13 Mathew NT, Meyer JS. Pathogenesis and natural history of transient global amnesia. Stroke 1974;5:303-11.

14 Woo M-H, Lee HS, Kim J. Effect of pioglitazone in acute ischemic stroke patients with diabetes mellitus: a nested case-control study. Cardiovasc Diabetol 2019;18:67.

$15 \mathrm{Kim} \mathrm{NH}$, Han $\mathrm{KH}$, Choi J, et al. Use of fenofibrate on cardiovascular outcomes in statin users with metabolic syndrome: propensity matched cohort study. BMJ 2019;366:I5125.

16 Seong SC, Kim Y-Y, Park SK, et al. Cohort profile: the National health insurance Service-National health screening cohort (NHIS-HEALS) in Korea. BMJ Open 2017;7:24

17 Higashida K, Okazaki S, Todo K, et al. A multicenter study of transient global amnesia for the better detection of magnetic resonance imaging abnormalities. Eur J Neurol 2020;27:2117-24.

18 Michel P, Beaud V, Eskandari A, et al. Ischemic amnesia: causes and outcome. Stroke 2017;48:2270-3.

19 Nitta Y, Tahara N, Tahara A, et al. Pioglitazone decreases coronary artery inflammation in impaired glucose tolerance and diabetes mellitus: evaluation by FDG-PET/CT imaging. JACC Cardiovasc Imaging 2013;6:1172-82.

20 Mangla A, Navi BB, Layton K, et al. Transient global amnesia and the risk of ischemic stroke. Stroke 2014;45:389-93.

21 Bartsch T, Deuschl G. Transient global amnesia: functional anatomy and clinical implications. Lancet Neurol 2010;9:205-14.

22 Austin PC. The use of propensity score methods with survival or time-to-event outcomes: reporting measures of effect similar to those used in randomized experiments. Stat Med 2014;33:1242-58.

23 Cattaino G, Querin F, Pomes A, et al. Transient global amnesia. Acta Neurol Scand 1984;70:385-90.

24 Romoli M, Tuna MA, McGurgan I, et al. Long-Term risk of stroke after transient global amnesia in two prospective cohorts. Stroke 2019;50:2555-7.

25 Garg A, Limaye K, Shaban A, et al. Transient global amnesia does not increase the risk of subsequent ischemic stroke: a propensity score-matched analysis. J Neurol 2021;268:3301-6.

26 Croft PB, Heathfield KW, Swash M. Differential diagnosis of transient amnesia. Br Med J 1973;4:593-6.

27 Arena JE, Brown RD, Mandrekar J, et al. Long-Term outcome in patients with transient global amnesia: a population-based study. Mayo Clin Proc 2017:92:399-405.

28 Romoli M, Tuna MA, Li L, et al. Time trends, frequency, characteristics and prognosis of short-duration transient global amnesia. Eur J Neurol 2020;27:887-93.

29 Himeno T, Kuriyama M, Takemaru M, et al. Vascular risk factors and internal jugular venous flow in transient global amnesia: a study of 165 Japanese patients. J Stroke Cerebrovasc Dis 2017;26:2272-8. 